\title{
Conversion surgery in patients with pancreatic cancer and peritoneal metastasis
}

\author{
Suguru Yamada ${ }^{1}$, Tsutomu Fujii ${ }^{2}$, Tomohisa Yamamoto ${ }^{3}$, Hideki Takami ${ }^{1}$, Isaku Yoshioka ${ }^{2}$, So Yamaki $^{3}$, \\ Fuminori Sonohara ${ }^{1}$, Kazuto Shibuya ${ }^{2}$, Fuyuhiko Motoi ${ }^{4}$, Satoshi Hirano ${ }^{5}$, Yoshiak Murakami ${ }^{6}$, \\ Hitoshi Inoue $^{7}$, Masamichi Hayashi ${ }^{1}$, Daisuke Hashimoto ${ }^{3}$, Kenta Murotani ${ }^{8}$, Joji Kitayama ${ }^{9}$, \\ Hideki Ishikawa $^{10}$, Yasuhiro Kodera ${ }^{1}$, Mitsugu Sekimoto ${ }^{3}$, Sohei Satoi ${ }^{3}$
}

${ }^{1}$ Nagoya University Graduate School of Medicine, Gastroenterological Surgery, Nagoya, Japan; ${ }^{2}$ Department of Surgery and Science, Faculty of Medicine, Academic Assembly, University of Toyama, Toyama, Japan; ${ }^{3}$ Department of Surgery, Kansai Medical University, Hirakata, Japan; ${ }^{4}$ Department of Surgery, Tohoku University Graduate School of Medicine, Sendai, Japan; ${ }^{5}$ Department of Surgery, Institute of Biomedical and Health Sciences, Hiroshima University, Hiroshima, Japan; ${ }^{6}$ Department of Gastroenterological Surgery II, Faculty of Medicine, Hokkaido University, Sapporo, Japan; ${ }^{7}$ Department of HBP and Breast Surgery, Ehime University Graduate School of Medicine, Ehime, Japan; ${ }^{8}$ Biostatistics Centre, Graduate School of Medicine, Kurume University, Fukuoka, Japan; 'Department of Gastrointestinal Surgery, Jichi Medical University, Tochigi, Japan; ${ }^{10}$ Department of Molecular-Targeting Cancer Prevention, Graduate School of Medical Science, Kyoto Prefectural University of Medicine, Kyoto, Japan

Contributions: (I) Conception and design: S Yamada, T Fujii, S Satoi; (II) Administrative support: J Kitayama, Y Kodera, M Sekimoto, S Satoi; (III) Provision of study materials or patients: T Yamamoto, H Takami, I Yoshioka, S Yamaki, F Sonohara, K Shibuya, F Motoi, S Hirano, Y Murakami, H Inoue, M Hayashi; (IV) Collection and assembly of data: T Yamamoto, F Sonohara, H Inoue; (V) Data analysis and interpretation: S Yamada, K Murotani; (VI) Manuscript writing: All authors; (VII) Final approval of manuscript: All authors.

Correspondence to: Prof. Sohei Satoi. Department of Surgery, Kansai Medical University, 2-5-1, Shin-machi, Hirakata 573-1010, Japan. Email: satoi@hirakata.kmu.ac.jp.

Background: Pancreatic ductal adenocarcinoma (PDAC) is among the most lethal malignancies globally. We have previously explored the clinical efficacy of intraperitoneal (IP) paclitaxel therapy for patients with PDAC and peritoneal metastasis, which demonstrated favourable response and disease control rates. However, the real implications of conversion surgery after IP therapy remain unclear.

Methods: We conducted two multicenter clinical trials of IP therapy with paclitaxel in patients with PDAC and peritoneal metastasis. We focused on patients who underwent conversion surgery and investigated the long-term outcomes, particularly, initial recurrence patterns and long-term survival.

Results: Seventy-nine patients with PDAC and peritoneal metastasis were treated, and 33 (41.8\%) patients received SP (intravenous IP paclitaxel with S-1) and 46 (58.3\%) were administered GAP (intravenous gemcitabine + nab-paclitaxel combined with IP paclitaxel) combination therapy. Of the 79 patients, 16 (20.3\%) underwent conversion surgery. The median time to surgery was 9.0 (range, 4.1-13.0) months after the initiation of chemotherapy. Finally, 13 (81.3\%) patients underwent R0 resection. Evans grade was IIA in nine patients, IIB in four patients, III in two patients, and IV in one patient. The median overall survival time in patients who underwent conversion surgery was 32.5 (range, 13.5-66.9) months. Twelve (75.0\%) patients were found to have experienced recurrence after conversion surgery. Especially, peritoneal recurrence was observed in $50 \%$ of patients as the initial recurrence pattern. The median recurrence-free survival time was 9.2 (range, 5.1-32.8) months, and three patients have survived without recurrence to date.

Conclusions: Our IP therapy displays promising clinical efficacy with acceptable tolerability in patients with PDAC and peritoneal metastasis. Although we could observe some super-responders in the cohort, further improvements in IP therapy are warranted. 
Keywords: Conversion surgery; pancreatic cancer; peritoneal metastasis; intraperitoneal therapy (IP therapy)

Submitted Jun 23, 2020. Accepted for publication Jul 16, 2020.

doi: 10.21037/jgo-20-243

View this article at: http://dx.doi.org/10.21037/jgo-20-243

\section{Introduction}

Pancreatic ductal adenocarcinoma (PDAC) is one of the most lethal malignancies worldwide. The median survival time (MST) of patients with distant metastasis is estimated to be less than 12 months (1). In particular, the presence of peritoneal metastasis can cause the development of massive ascites and intestinal obstruction, leading to malnutrition and poor performance status, which could deprive patients of the opportunity to receive chemotherapy $(2,3)$. Peritoneal metastasis is generally treated with systemic chemotherapy in the same manner as other distant metastases. However, intraperitoneal (IP) chemotherapy appears advantageous for treating peritoneal dissemination because of the high drug concentration that is achievable in the peritoneal cavity, which is in direct contact with tumour nodules, compared with the effects of systemic chemotherapy (4-9).

To treat this dismal disease, we previously explored the clinical efficacy of IP paclitaxel therapy in patients with PDAC and peritoneal metastasis, demonstrating favourable response and disease control rates. In our studies, the MST and 1-year overall survival rate were 14.5-16.3 months and 61-62\%, respectively, with conversion surgery performed in $17.4-24.2 \%$ of the enrolled patients (10). Concerning overall survival, the patients who underwent conversion surgery survived significantly longer than those who did not.

However, the real implications of conversion surgery after IP therapy in patients with PDAC and peritoneal metastasis are still unclear because of the lack of clinical experience in this therapy. Therefore, we focused on patients who underwent conversion surgery and investigated long-term outcomes including initial recurrence patterns and long-term survival. We present the following article in accordance with the STROBE reporting checklist (available at http://dx.doi.org/10.21037/jgo-20-243).

\section{Methods}

\section{Enrolled patients}

We conducted two multicenter clinical trials of IP therapy with paclitaxel in patients with PDAC and peritoneal metastasis. In the first trial, intravenous (IV) and IP paclitaxel with S-1 (SP) were tested as combination therapy (10), and IV gemcitabine + nab-paclitaxel combined with IP paclitaxel (GAP) was subsequently evaluated in the second trial (11).

The eligibility criteria in both trials were briefly as follows: histologically or cytologically proven PDAC; peritoneal metastasis in patients with otherwise resectable cancer, or the presence of cancer cells in patients with unresectable locally advanced cancer; no prior receipt of chemotherapy, or chemotherapy started within 2 months; Eastern Cooperative Oncology Group performance status of 0 or 1; adequate bone marrow, liver, and renal function; and age $\geq 20$ years and $<80$ years $(10,11)$.

The exclusion criteria were as follows: presence of distant metastasis excluding the ovaries; contraindication for S-1, gemcitabine, nab-paclitaxel, or paclitaxel; massive ascites; bleeding in the alimentary tract with repetitive blood transfusion; other active concomitant malignancies; and invasion of more than half of the alimentary tract by the primary tumour or peritoneal deposits. These studies were conducted in accordance with the Declaration of Helsinki (as revised in 2013), and the study protocol was approved by the institutional review board of the affiliated hospital (UMIN000009446) (UMIN000018878) and informed consent was taken from all individual participants.

\section{Treatment}

If peritoneal dissemination or positive peritoneal cytology was detected during staging laparoscopy or open laparotomy, a peritoneal access port was implanted in the lower abdomen. In the former SP trial, S-1 was orally administered twice daily at a dose of $80 \mathrm{mg} / \mathrm{m}^{2} / \mathrm{d}$ for 14 consecutive days, followed by 7 days of rest. Paclitaxel was administered intravenously at a dose of $50 \mathrm{mg} / \mathrm{m}^{2}$ and intraperitoneally at $20 \mathrm{mg} / \mathrm{m}^{2}$ on days 1 and 8 . Whereas in the latter GAP trial, IV nab-paclitaxel combined with gemcitabine was administered combined with IP paclitaxel on days 1,8 , and 15 , followed by 1 week of rest. The 
Table 1 Patient characteristics at diagnosis

\begin{tabular}{ll}
\hline Characteristics & Value \\
\hline Age, years & $69(47.0-79)$ \\
Sex, male/female & $40(50.6) / 39(49.4)$ \\
BMl, kg/m² & $20.3(13.3-30.5)$ \\
Performance status, 0/1 & $55(69.6) / 24(30.4)$ \\
Tumor location, head/body and tail & $22(27.8) / 57(72.2)$ \\
Tumor size, mm & $37(18-105)$ \\
Resectability, R/BR/UR & $12(15.2) / 25(31.6) / 42(53.2)$ \\
Ascites, -/+ & $34(43.0) / 45(57.0)$ \\
Peritoneal dissemination, -/+ & $28(35.4) / 51(64.6)$ \\
Peritoneal (washing) cytology, $-/+$ & $1(1.3) / 78(98.7)$ \\
Albumin, g/dL & $3.7(2.5-4.8)$ \\
CA19-9 level, U/mL & $539(0.9-38,000)$ \\
CA125 level, U/mL & $43.9(9-385.4)$ \\
Biliary drainage, -/+ & $65(82.3) / 14(17.7)$ \\
Duration of protocol therapy, & $7.1(0-22.6)$ \\
months & $33(41.8) / 46(58.3)$ \\
Regimen, SP/GAP & \\
\hline
\end{tabular}

Data are presented as the median (range) or $\mathrm{n}$ (\%). BMI, body mass index; CA19-9, carbohydrate antigen 19-9; CA125, carbohydrate antigen 125; R, resectable; BR, borderline resectable; UR, unresectable; SP, S-1 + paclitaxel; GAP, gemcitabine + nab-paclitaxel + paclitaxel.

treatment course was repeated until unacceptable toxicity, disease progression, or surgery. The criteria for surgical resection (defined as conversion surgery) were as follows: Eastern Cooperative Oncology Group performance status of 0 or 1 ; marked tumour shrinkage; decreased or normalisation of tumour marker levels; washing cytology via peritoneal access port turned negative (twice in a row); and disappearance of peritoneal deposits on staging laparoscopy. The decision to proceed to conversion surgery was based on an interval exceeding 8 months between the initial treatment and surgical resection (12).

\section{Statistical analysis}

All statistical analyses were performed using JMP Pro version 14.2.0 (SAS Institute, Cary, NC, USA). Continuous variables were expressed as the median (range). Overall survival was defined as the time from treatment introduction to all-cause death. Recurrence-free survival was defined as the time from conversion surgery to diagnosis of recurrence.

\section{Results}

\section{Patient recruitment}

A total of 79 patients with pancreatic cancer and peritoneal metastasis were treated in our two studies that used IP therapy with paclitaxel. Among them, 33 (41.8\%) patients received SP (IV and IP paclitaxel with S-1) and 46 (58.3\%) underwent GAP (IV gemcitabine + nab-paclitaxel combined with IP paclitaxel) combination therapy.

\section{Patient characteristics}

Patient characteristics of 79 cases are shown in Table 1. The tumour was located at the pancreatic head in 22 $(27.8 \%)$ patients and at the pancreatic body/tail in 57 (72.2\%) patients, and the median tumour diameter was 37 (range, 18-105) mm. Primary tumours were categorised as resectable in $12(15.2 \%)$ patients, borderline resectable in $25(31.6 \%)$ patients, and unresectable and locally advanced in 42 (53.2\%) patients (13), based on the National Comprehensive Cancer Network (NCCN) guidelines. Malignant ascites was observed in 45 (57.0\%) patients upon laparoscopy or laparotomy, and peritoneal dissemination was confirmed in $51(64.6 \%)$ patients. The median treatment duration was 7.1 (range, 0-22.6) months (Table 1).

\section{Conversion surgery}

Of the 79 patients, $16(20.3 \%)$ patients underwent conversion surgery, and their clinical characteristics are shown in Table 2. The tumour location in $13(81.3 \%)$ patients was the pancreatic body and tail. Eleven (68.8\%) patients had peritoneal dissemination at diagnosis, and five patients had positive peritoneal washing cytology plus unresectable locally advanced cancer preoperatively. The median time to surgery was 9.0 (range, 4.1-13.0) months after the initiation of chemotherapy. Finally, 13 (81.3\%) patients underwent R0 resection. Evans grade was IIA in nine patients, IIB in four patients, III in two patients, and IV in one patient.

\section{Survival outcomes}

The median overall survival time of the patients who 


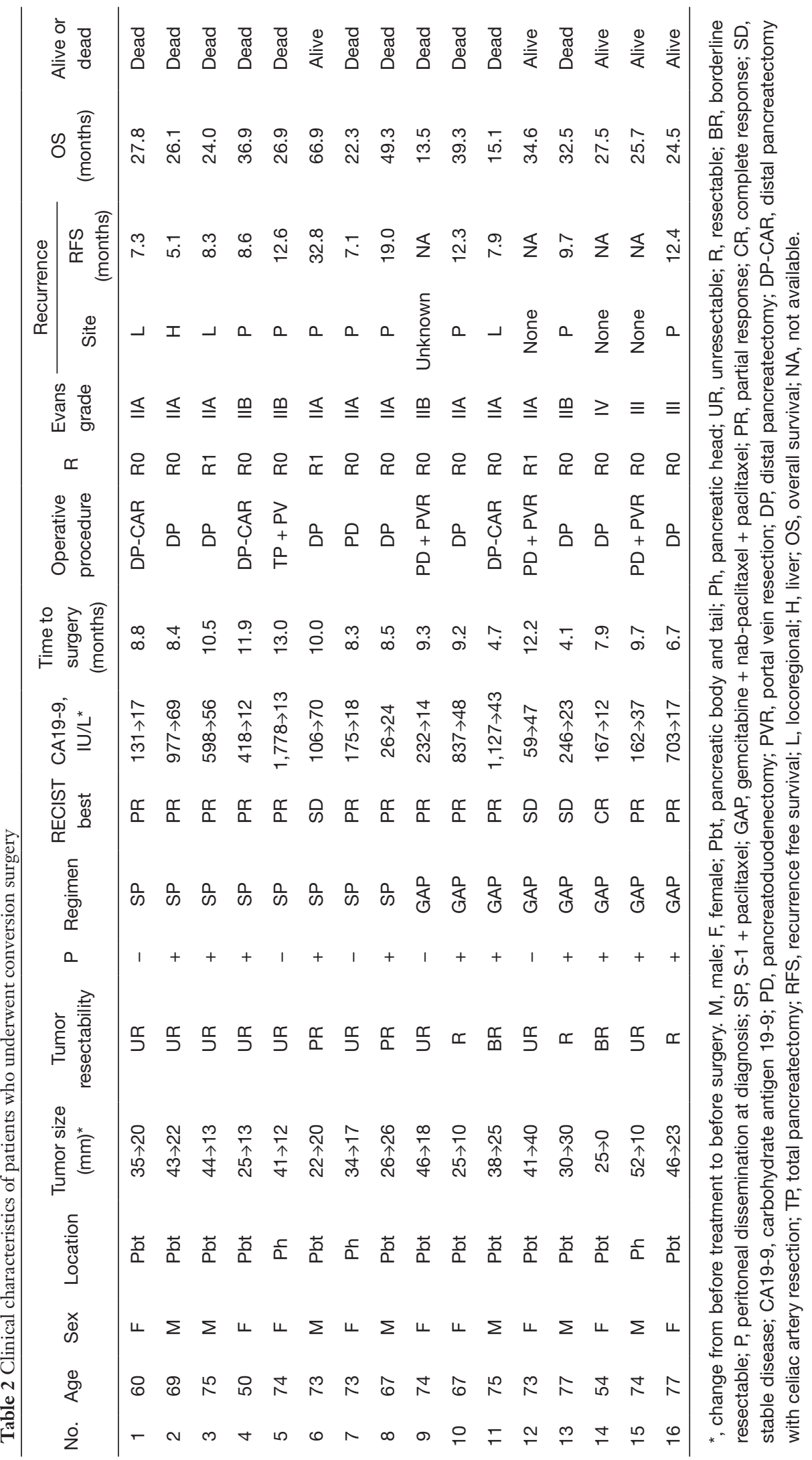



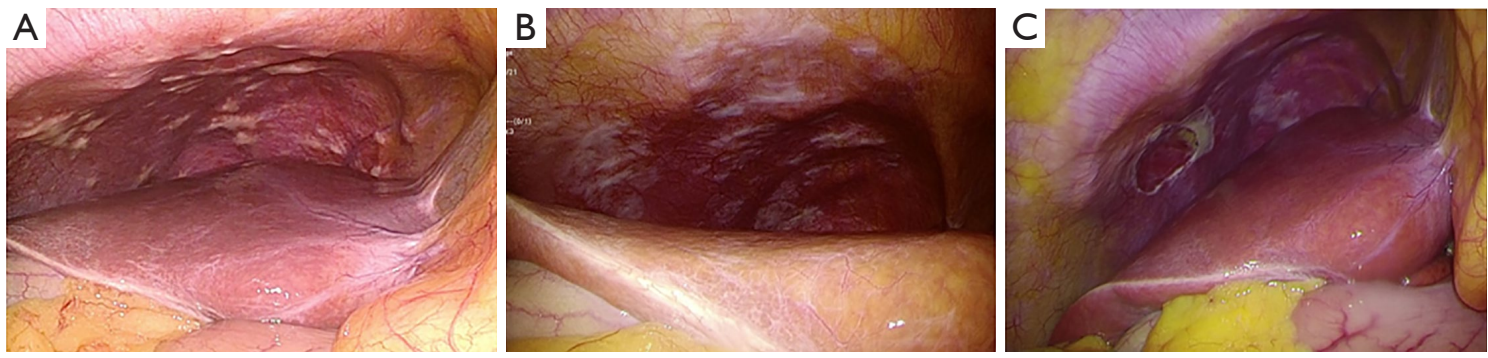

Figure 1 Intra-abdominal findings on diagnostic laparoscopy. (A) Multiple peritoneal deposits were observed in the right subphrenic space before treatment introduction. (B) Withdrawal of peritoneal deposits was confirmed at second diagnostic laparoscopy, and pathological examination showed no evidence of malignancy. (C) Intra-abdominal findings at the time of conversion surgery.
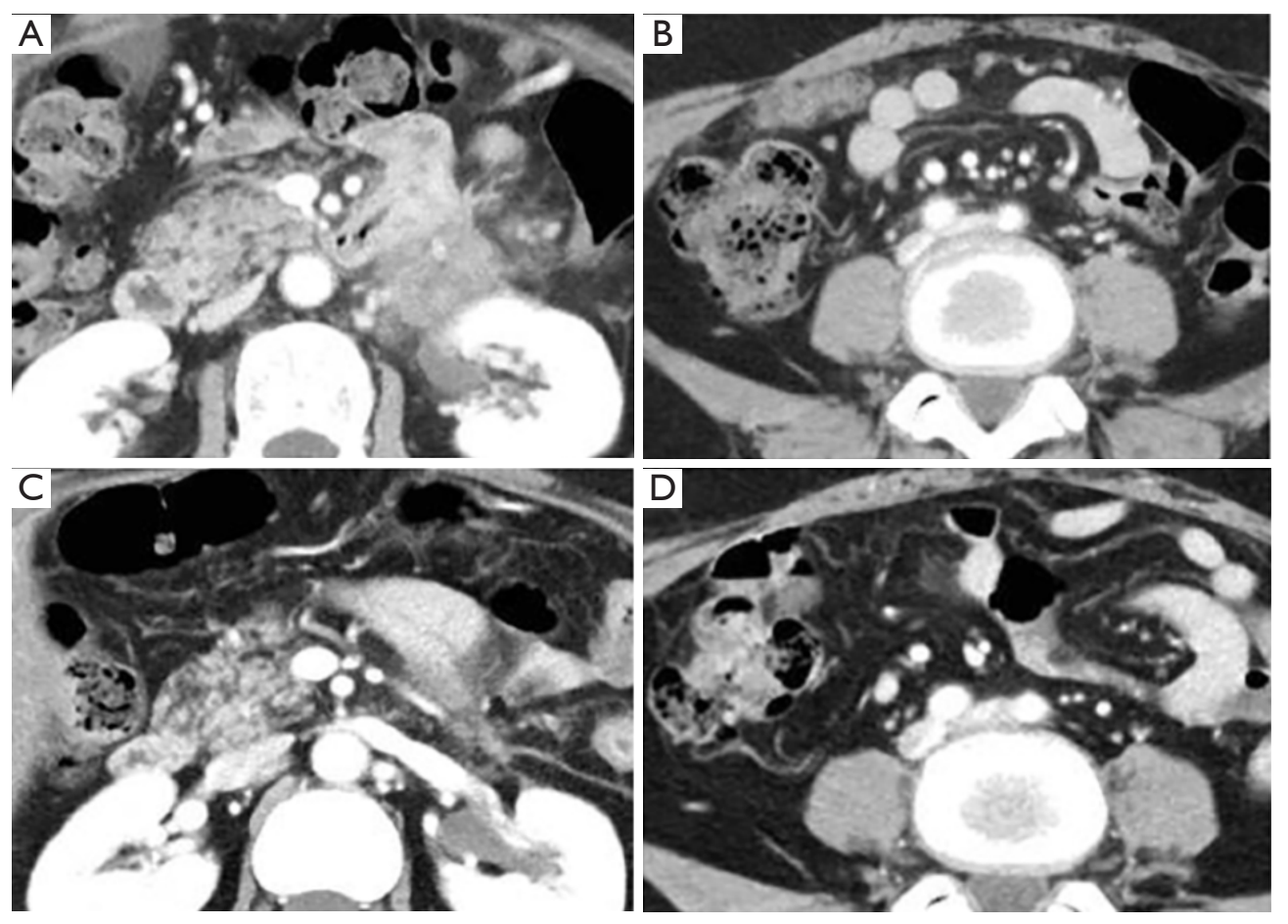

Figure 2 Computed tomography findings during treatment. (A) The tumour located in pancreatic tail was observed before treatment introduction. (B) The peritoneal deposits were also found in peritoneal cavity. (C) The tumour shrinkage following treatment was confirmed. (D) The disappearance of peritoneal deposits was observed.

underwent conversion surgery was 32.5 (range, 13.5-66.9) months. After conversion surgery, a total of $12(75.0 \%)$ patients experienced recurrence. Especially, peritoneal recurrence was observed in $50 \%$ of patients as the initial recurrence pattern. The median recurrence-free survival time was 9.2 (range, 5.1-32.8) months, and three patients have survived without recurrence thus far.
Among the patients, case no. 14 was successfully cured. GAP therapy was introduced in this 54-year old female patient because she was diagnosed as having PDAC with peritoneal metastasis. During her 8-month treatment, the disappearance of peritoneal deposits was confirmed pathologically (Figures 1-3). Finally, she underwent distal pancreatectomy as conversion surgery, and surprisingly, the 

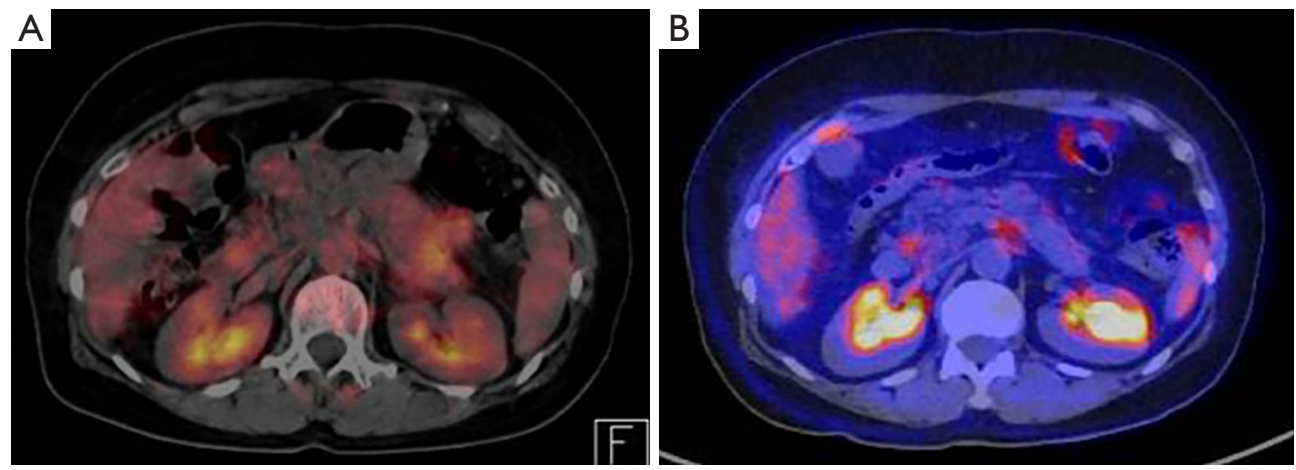

Figure 3 Positron emission tomography findings during treatment. (A) Standardized uptake value on the pancreatic tail was observed before treatment introduction. (B) There was no uptake in the area.

final pathological finding showed Evans grade IV.

\section{Discussion}

IP chemotherapy enables peritoneal deposits to be exposed to high concentrations of drugs without increasing the blood concentration, which is considered to be advantageous (14). Further, the effective duration after IP administration is determined by the molecular characteristics of the drugs; in that sense, paclitaxel is suitable for use (4). In the area of gastroenterological malignancies, IV/IP paclitaxel + S-1 therapy in gastric cancer and peritoneal metastasis was evaluated in a phase I/II study $(6,14)$, and the phase III PHOENIX-GC trial was conducted to compare this regimen with standard therapy (15). We also reported the promising clinical efficacy and acceptable tolerability of IP paclitaxel therapy in PDAC and peritoneal metastasis patients $(10,11)$.

In general, most patients with PDAC and peritoneal metastasis exhibit massive ascites and a subsequent poor performance status, leading to fewer opportunities to receive chemotherapy (3). Surprisingly, a previous report observed considerably poor survival following weekly paclitaxel in patients with PDAC and malignant ascites (16). More recently, the MST in patients with PDAC and peritoneal dissemination was reported to be only 7 months, and that in patients with locally advanced disease with positive peritoneal washing cytology was 6 months (17). Our previous report also revealed MSTs of 8 months in patients with PDAC and peritoneal metastasis and 13 months in patients with locally advanced disease and positive peritoneal washing cytology (3). Considering that patients with peritoneal metastasis generally have an extremely poor prognosis, the results of our studies are encouraging.

Recently, multidisciplinary treatment combining chemoor chemoradiotherapy and subsequent surgery has been widely accepted and regarded as a promising strategy. Generally, the rate of conversion surgery after induction therapy in locally advanced PDAC ranged from $1.3-36 \%$, and the MST after resection has been reported to reach 18.2-41.8 months (18-25). Also, Suker et al. conducted a systematic review that showed the ratio of conversion surgery was $28 \%$, however; the long-term survival outcomes remain unconfirmed (26).

In metastatic disease, the rate of conversion surgery is even lower. Inherently, few studies regarding this disease have been reported, in which the conversion ratio ranged from $2-4.5 \%(27,28)$. That is, the conversion ratio of the intention-to-treat population remained below $5 \%$. Notably, there has been no report focused on peritoneal metastasis. In our study, the combination therapy enabled $17.4-24.2 \%$ patients to be eligible for conversion surgery, a considerable achievement given the generally poor outcomes of patients with PDAC and peritoneal metastasis. Although our therapy had a marked impact, most patients who underwent conversion surgery unfortunately recurred. However, we observed some super-responders in the cohort, and thus further improvement of IP therapy is warranted.

In conclusion, our IP therapy displayed promising clinical efficacy with acceptable tolerability in patients with PDAC and peritoneal metastasis. However, these studies were conducted as a phase I/II study with a single-arm design. Therefore, we have launched a phase III study to compare survival outcomes between this IP therapy and 
standard chemotherapy.

\section{Acknowledgments}

We would like to express our sincere appreciation to SY, HR, NK, KU, YN, AT, AM, YN, KA, MW, TA, KW, MY, YT, GH, HS, YM, SS, FM, and NI from the Japan study group of pancreatic ductal adenocarcinoma with peritoneal metastasis for their significant contributions to this study. We also thank H. Nikki March, PhD, from Edanz Group (https://en-author-services.edanzgroup.com/) for editing a draft of this manuscript.

Funding: None.

\section{Footnote}

Provenance and Peer Review: This article was commissioned by the Guest Editors (Paul H. Sugarbaker and Kurt Van der Speeten) for the focused issue "Intraperitoneal Chemotherapy for Peritoneal Metastases: HIPEC, EPIC, NIPEC, PIPAC and More" published in fournal of Gastrointestinal Oncology. This article has undergone external peer review.

Reporting Checklist: The authors have completed the STROBE reporting checklist. Available at http://dx.doi. org/10.21037/jgo-20-243

Conflicts of Interest: All authors have completed the ICMJE uniform disclosure form (available at http://dx.doi. org/10.21037/jgo-20-243). The focused issue was sponsored by the Peritoneal Surface Oncology Group International (PSOGI). The authors have no other conflicts of interest to declare.

Ethical Statement: The authors are accountable for all aspects of the work in ensuring that questions related to the accuracy or integrity of any part of the work are appropriately investigated and resolved. These studies were conducted in accordance with the Declaration of Helsinki (as revised in 2013), and the study protocol was approved by the institutional review board of the affiliated hospital (UMIN000009446) (UMIN000018878) and informed consent was taken from all individual participants.

Open Access Statement: This is an Open Access article distributed in accordance with the Creative Commons Attribution-NonCommercial-NoDerivs 4.0 International
License (CC BY-NC-ND 4.0), which permits the noncommercial replication and distribution of the article with the strict proviso that no changes or edits are made and the original work is properly cited (including links to both the formal publication through the relevant DOI and the license). See: https://creativecommons.org/licenses/by-nc-nd/4.0/.

\section{References}

1. Thomassen I, Lemmens VE, Nienhuijs SW, et al. Incidence, prognosis, and possible treatment strategies of peritoneal carcinomatosis of pancreatic origin: a population-based study. Pancreas 2013;42:72-5.

2. Takahara N, Isayama H, Nakai $Y$, et al. Pancreatic cancer with malignant ascites: clinical features and outcomes. Pancreas 2015;44:380-5.

3. Satoi S, Yanagimoto H, Yamamoto T, et al. A clinical role of staging laparoscopy in patients with radiographically defined locally advanced pancreatic ductal adenocarcinoma. World J Surg Oncol 2016;14:14.

4. Markman M, Brady MF, Spirtos NM, et al. Phase II trial of intraperitoneal paclitaxel in carcinoma of the ovary, tube, and peritoneum: a Gynecologic Oncology Group Study. J Clin Oncol 1998;16:2620-4.

5. Armstrong DK, Bundy B, Wenzel L, et al. Intraperitoneal cisplatin and paclitaxel in ovarian cancer. N Engl J Med 2006;354:34-43.

6. Ishigami H, Kitayama J, Kaisaki S, et al. Phase II study of weekly intravenous and intraperitoneal paclitaxel combined with S-1 for advanced gastric cancer with peritoneal metastasis. Ann Oncol 2010;21:67-70.

7. Kodera Y, Imano M, Yoshikawa T, et al. A randomized phase II trial to test the efficacy of intra-peritoneal paclitaxel for gastric cancer with high risk for the peritoneal metastasis (INPACT trial). Jpn J Clin Oncol 2011;41:283-6.

8. Yamaguchi H, Kitayama J, Ishigami H, et al. A phase 2 trial of intravenous and intraperitoneal paclitaxel combined with S-1 for treatment of gastric cancer with macroscopic peritoneal metastasis. Cancer 2013;119:3354-8.

9. Takahara N, Isayama H, Nakai $Y$, et al. Intravenous and intraperitoneal paclitaxel with S-1 for refractory pancreatic cancer with malignant ascites: an interim analysis. J Gastrointest Cancer 2014;45:307-11.

10. Satoi S, Fujii T, Yanagimoto H, et al. Multicenter Phase II Study of Intravenous and Intraperitoneal Paclitaxel With S-1 for Pancreatic Ductal Adenocarcinoma Patients With Peritoneal Metastasis. Ann Surg 2017;265:397-401. 
11. Yamada S, Fujii T, Yamamoto T, et al. Phase I/II study of adding intraperitoneal paclitaxel in patients with pancreatic cancer and peritoneal metastasis. Br J Surg 2020;107:1811-7.

12. Satoi S, Yamaue H, Kato K, et al. Role of adjuvant surgery for patients with initially unresectable pancreatic cancer with a long-term favorable response to non-surgical anti-cancer treatments: results of a project study for pancreatic surgery by the Japanese Society of HepatoBiliary-Pancreatic Surgery. J Hepatobiliary Pancreat Sci 2013;20:590-600.

13. Network NCC. NCCN clinical practice guidelines in oncology (NCCN Guidelines). Pancreatic adenocarcinoma. NCCN; 2016.

14. Ishigami H, Kitayama J, Otani K, et al. Phase I pharmacokinetic study of weekly intravenous and intraperitoneal paclitaxel combined with S-1 for advanced gastric cancer. Oncology 2009;76:311-4.

15. Ishigami H, Fujiwara Y, Fukushima R, et al. Phase III Trial Comparing Intraperitoneal and Intravenous Paclitaxel Plus S-1 Versus Cisplatin Plus S-1 in Patients With Gastric Cancer With Peritoneal Metastasis: PHOENIX-GC Trial. J Clin Oncol 2018;36:1922-9.

16. Shukuya T, Yasui H, Boku N, et al. Weekly Paclitaxel after failure of gemcitabine in pancreatic cancer patients with malignant ascites: a retrospective study. Jpn J Clin Oncol 2010;40:1135-8.

17. Ferrone CR, Haas B, Tang L, et al. The influence of positive peritoneal cytology on survival in patients with pancreatic adenocarcinoma. J Gastrointest Surg 2006;10:1347-53.

18. Mukherjee S, Hurt CN, Bridgewater J, et al. Gemcitabinebased or capecitabine-based chemoradiotherapy for locally advanced pancreatic cancer (SCALOP): a multicentre, randomised, phase 2 trial. Lancet Oncol 2013;14:317-26.

19. Youl M, Hashem S, Brade A, et al. Induction gemcitabine plus concurrent gemcitabine and radiotherapy for locally advanced unresectable or resected pancreatic cancer. Clin
Oncol (R Coll Radiol) 2014;26:203-9.

20. Marthey L, Sa-Cunha A, Blanc JF, et al. FOLFIRINOX for locally advanced pancreatic adenocarcinoma: results of an AGEO multicenter prospective observational cohort. Ann Surg Oncol 2015;22:295-301.

21. Sadot E, Doussot A, O'Reilly EM, et al. FOLFIRINOX Induction Therapy for Stage 3 Pancreatic Adenocarcinoma. Ann Surg Oncol 2015;22:3512-21.

22. Hammel P, Huguet F, van Laethem JL, et al. Effect of Chemoradiotherapy vs Chemotherapy on Survival in Patients With Locally Advanced Pancreatic Cancer Controlled After 4 Months of Gemcitabine With or Without Erlotinib: The LAP07 Randomized Clinical Trial. JAMA 2016;315:1844-53.

23. Gemenetzis G, Groot VP, Blair AB, et al. Survival in Locally Advanced Pancreatic Cancer After Neoadjuvant Therapy and Surgical Resection. Ann Surg 2019;270:340-7.

24. Maggino L, Malleo G, Marchegiani G, et al. Outcomes of Primary Chemotherapy for Borderline Resectable and Locally Advanced Pancreatic Ductal Adenocarcinoma. JAMA Surg 2019;154:932-42.

25. Satoi S, Yamamoto T, Yamaki S, et al. Surgical indication for and desirable outcomes of conversion surgery in patients with initially unresectable pancreatic ductal adenocarcinoma. Ann Gastroenterol Surg 2019;4:6-13.

26. Suker M, Beumer BR, Sadot E, et al. FOLFIRINOX for locally advanced pancreatic cancer: a systematic review and patient-level meta-analysis. Lancet Oncol 2016;17:801-10.

27. Wright GP, Poruk KE, Zenati MS, et al. Primary Tumor Resection Following Favorable Response to Systemic Chemotherapy in Stage IV Pancreatic Adenocarcinoma with Synchronous Metastases: a Bi-institutional Analysis. J Gastrointest Surg 2016;20:1830-5.

28. Frigerio I, Regi P, Giardino A, et al. Downstaging in Stage IV Pancreatic Cancer: A New Population Eligible for Surgery? Ann Surg Oncol 2017;24:2397-403.
Cite this article as: Yamada S, Fujii T, Yamamoto T, Takami H, Yoshioka I, Yamaki S, Sonohara F, Shibuya K, Motoi F, Hirano S, Murakami Y, Inoue H, Hayashi M, Hashimoto D, Murotani K, Kitayama J, Ishikawa H, Kodera Y, Sekimoto M, Satoi S. Conversion surgery in patients with pancreatic cancer and peritoneal metastasis. J Gastrointest Oncol 2021;12 (Suppl 1):S110-S117. doi: 10.21037/jgo-20-243 